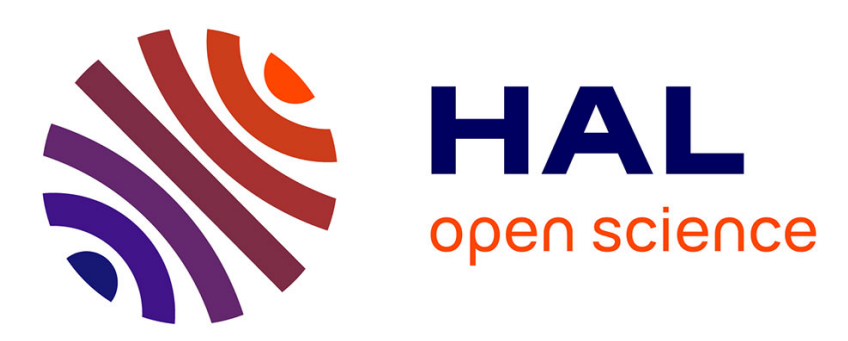

\title{
Towards a model for the multidimensional analysis of field data
}

\author{
S. Bimonte, Myoung-Ah Kang
}

\section{To cite this version:}

S. Bimonte, Myoung-Ah Kang. Towards a model for the multidimensional analysis of field data. 14th East-European Conference on Advances in Databases and Information Systems, ADBIS 2010, Sep 2010, Novi Sad, Serbia. 15 p. hal-00527729

\section{HAL Id: hal-00527729 \\ https://hal.science/hal-00527729}

Submitted on 14 Dec 2010

HAL is a multi-disciplinary open access archive for the deposit and dissemination of scientific research documents, whether they are published or not. The documents may come from teaching and research institutions in France or abroad, or from public or private research centers.
L'archive ouverte pluridisciplinaire HAL, est destinée au dépôt et à la diffusion de documents scientifiques de niveau recherche, publiés ou non, émanant des établissements d'enseignement et de recherche français ou étrangers, des laboratoires publics ou privés. 


\title{
Towards a Model for the Multidimensional Analysis of Field Data
}

\author{
Sandro Bimonte ${ }^{1}$, Myoung-Ah Kang ${ }^{2}$ \\ ${ }^{1}$ CEMAGREF, Campus des CEZEAUX, \\ 63173 AUBIERE, France \\ ${ }^{2}$ LIMOS-UMR CNRS 6158,ISIMA, Blaise Pascal University,Campus des CEZEAUX, \\ 63173 AUBIERE, France \\ ${ }^{1}$ Sandro.bimonte@cemagref.fr, ${ }^{2}$ kang@isima.fr
}

\begin{abstract}
Integration of spatial data into multidimensional models leads to the concept of Spatial OLAP (SOLAP). Usually, SOLAP models exploit discrete spatial data. Few works integrate continuous field data into dimensions and measures. In this paper, we provide a multidimensional model that supports measures and dimension as continuous field data, independently of their implementation.
\end{abstract}

Keywords: Spatial OLAP, Field data, Spatial Data Warehouses, Multidimensional models

\section{Introduction}

It has been estimated that about $80 \%$ of the data stored in corporate databases integrates geographic information [7]. This information is typically represented according two models, depending on the nature of data: discrete (vector) and field [22]. The latter model represents the space as a continuous field. Fields have to be discretized to be represented into computers according to data input, and data analysis. These representations can be grouped into two categories: incomplete and complete. Incomplete representations store only some points and need supplementary functions to calculate the field in non-sampled areas. Complete representations associate estimated values to regions and assume that this value is valid for each point in the regions (raster). Fields are very adapted for modeling spatial phenomena such as pollution, temperature, etc. They allow a point by point analysis through the Map Algebra operators [16] [22].

In order to benefit from Data warehousing and OLAP decision support technologies [11] also in the context of spatial data, some works extended them leading to the concept of Spatial OLAP (SOLAP), which integrates OLAP and Geographic Information Systems (GIS) functionalities into a unique framework [2]. As for the model underlying SOLAP systems, several research issues from theoretical and implementation point of view have risen. Indeed, several works focus on indexing [20] and visualization techniques [6]. Motivated by the relevance of a formal 
representation of SOLAP data and operators, some spatio-multidimensional models based on vector data have been defined (a review can be found in [4]). Few works consider field data into multidimensional models [1], [23] and [15]. These models present some limitations that do not allow fully exploiting continuous field data into OLAP from these points of view: aggregation functions, hierarchies based on field, and independence of field data implementation. Thus, in this paper we propose a spatio-multidimensional model integrating field data.

The reminder of this paper is organized as follows: Section 2 recalls fundamentals of spatial analysis techniques, and existing SOLAP models for field data. Our model is proposed in the Section 3. Finally, in Section 4 conclusions and future work are presented.

\section{Related work}

The term Map Algebra was first introduced in [22] to describe operators on raster data (complete field data). Map Algebra operators are classified according to the number of grids and cells involved. Local operators apply a mathematical or logical function to the input grids, cell by cell. Focal operators calculate the new value of each cell using neighboring cells values of the input grid. Zonal operators calculate new values as a function of the values of the input grid which are associated with the zone of another grid, called zone layer. An extension of the Map Algebra (Cubic Map Algebra) to the temporal dimension is presented in [16]. The authors redefine Map Algebra operators on a cube of cells whose coordinates are three-dimensional. Then, the sets of operators are modified accordingly as shown on Figure 1. In [5] the authors define an algebraic model for field data. This framework provides a formal definition of Map Algebra operators independent of their implementation. Along this line, the work of [8] formally describes how Map Algebra fundamentals can be used for image manipulation. Finally, Map Algebra has been defined also for incomplete field data such as Voronoi tessellations [13]. Indeed, "Map Algebra obliges analysts to organize reality according to a particular data structure (raster) instead of allowing making the reality suggest the most adequate data structure for the analysis" [10].

On the other hand, the integration of spatial data into data warehousing and OLAP systems leads to the concept of Spatial OLAP (SOLAP). SOLAP is based on the spatial multidimensional model that defines spatial dimensions and spatial measures. In particular, a spatial dimension is a classical dimension whose levels contain spatial attributes. This allows for visualizing measures on maps to discover (spatial) relations and unknown patterns. According to the vector model, a spatial measure is defined as a set of geometries and/or the result of spatial operators [2] [14]. Some spatial multidimensional models, based on the vector model, have been proposed [4]. Despite of the significant analysis power of field data and its associated spatial analysis operators, only few works address this issue. [15] extends the concepts of spatial dimension to informally define "spatial matrix (raster) dimension" where at least one level represents raster data. Then, a member is a cell of the raster. Moreover, she introduces also the concept of "matrix cube" where each fact is associated to a cell of the raster data with its attributes. Aggregation functions are Map Algebra operators 
(local, focal and zonal). The author limits field data to raster. By this way, the work loses in terms of abstraction for field data. Indeed, Map Algebra has been defined also on other forms of representation of field data such as Voronoi tessellation [13], and terrain representations, such as TIN, that could be very useful for understanding and analyzing multidimensional data [6].

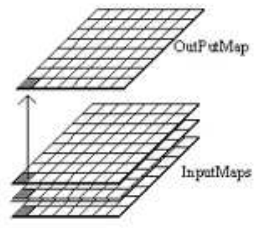

(a)

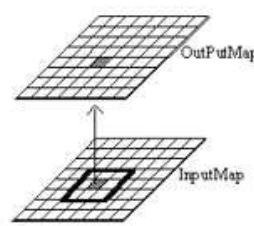

(b)

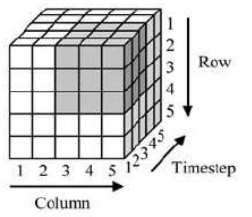

(d)

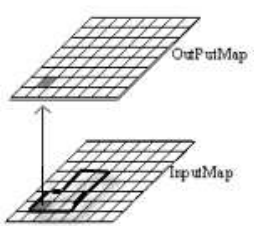

(c)

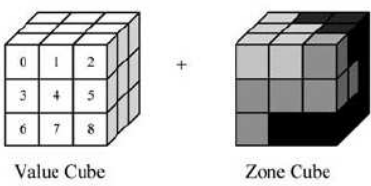

(e)

Figure 1. Map Algebra: a) local, b) focal, c) zonal [22], Cubic Map Algebra: d) focal, e) zonal [16].

Hence, [23] proposes a conceptual multidimensional model for taking into account field data independently of their implementation. They define a "field measure" as a value that changes in space and/or time. The model limits aggregation functions for field measure to local Map Algebra functions. They also introduce a "field hierarchy" when a level is a field data that is not linked to facts. Consequently, it is not possible to have field measures at different granularities, which are mandatory for spatial analysis [21]. Finally [1] defines the concept of "continuous cube" when spatial dimensions are composed of infinite spatial members whose associated numerical measures are calculated using interpolation functions, as they use an incomplete representation of spatial members. This model does not allow introduce field data as measures and in hierarchies.

Therefore, a formal model is necessary to create the foundation for a framework for the multidimensional analysis of field data as dimensions (field hierarchies) and measures. Table 1 shows the requirements for this model and how existing works address them. As shown in table 1, there is no existing model that supports all of these requirements. This is the reason why we propose a new SOLAP model in this paper. 
Table 1: Requirements for spatial multidimensional model for continuous field data.

\begin{tabular}{|c|c|c|c|}
\hline Requirements & {$[1]$} & {$[23]$} & {$[15]$} \\
\hline Measures as continuous field data & NO & YES & $\begin{array}{c}\text { Partially (only for raster } \\
\text { data) }\end{array}$ \\
\hline Hierarchy on continuous field data & NO & NO & $\begin{array}{c}\text { Partially (only for raster } \\
\text { data) }\end{array}$ \\
\hline $\begin{array}{c}\text { Aggregation functions as Map Algebra } \\
\text { functions }\end{array}$ & NO & $\begin{array}{c}\text { Partially (only local } \\
\text { functions) }\end{array}$ & $\begin{array}{c}\text { Partially (only for raster } \\
\text { data) }\end{array}$ \\
\hline Independence of implementation & YES & YES & NO \\
\hline
\end{tabular}

\section{Spatio-multidimensional model for field data}

Before introducing our model we present a SOLAP application for monitoring earthquakes in Italian regions. Spatial analyst wants answer to queries like this: "Where were earthquakes, and what was their intensity per region at different scales (resolutions)?". This SOLAP application presents a measure that is a field object representing the earthquakes, a temporal dimension, and a spatial dimension that represents terrain models of Italian regions at different scales.

\subsection{Geographic data model}

In this section, we provide a uniform representation for field and vector data, which are used by the multidimensional model to define measures and dimensions members.

An Object represents an object of the real world described by some alphanumeric attributes. It is used in the model to represent levels and members (Sec. 3.2).

\section{Definition 1. Object}

An Object Structure $S_{e}$ is a tuple $\left\langle a_{1}, \ldots a_{n}\right\rangle$ where $\forall i \in[1, \ldots n] a_{i}$ is an attribute defined on a domain dom $\left(a_{i}\right)$

An Instance of an Object Structure $S_{e}$ is a tuple $\left\langle\operatorname{val}\left(a_{1}\right), \ldots\right.$ val $\left.\left(a_{n}\right)\right\rangle$ where $\forall i \in[1, \ldots n]$ $\operatorname{val}\left(a_{i}\right) \in \operatorname{dom}\left(a_{i}\right)$

We denote by 'I $\left(S_{e}\right)^{\prime}$ the set of instances of $S_{e}$

A Geographic Object extends an Object to represent geographic information according to the vector model. Indeed, a Geographic Object [3] is a geometry (geom) and an optional set of alphanumeric attributes $\left(\left[a_{1}, \ldots a_{n}\right]\right)$ whose values are associated to the whole geometry according to the vector model (Figure 2a).

\section{Definition 2. Geographic Object}

Let $g \subset R^{2}$ i.e. a subset of the Euclidian space. An Object Structure $S_{e}=$ Lgeom, $\left[a_{1}\right.$, $\left.\left.\ldots a_{n}\right]\right\rangle$ is a Geographic Object Structure if the domain of the attribute geom is a set of geometries: dom(geom) $\in 2^{g}$

geom is called 'geometric support' 


\section{Example 1.}

The geographic object structure representing Italian regions is $\mathrm{S}_{\text {region }}=\langle$ geom, name $\rangle$ where 'geom' is the geometric support, and 'name' is the name of the region. An instance of $S_{\text {region }}$ is $t_{2}=\left\langle p_{l o}\right.$, Lombardia $\rangle$ where ' $p_{\text {lo }}$ ' is the geometry of the region Lombardia (Figure 2a).

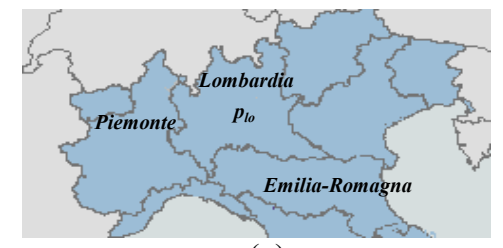

(a)

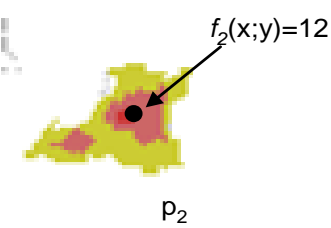

(b)

Figure 2: a) Italian regions: Instances of $\left.\mathrm{S}_{\text {region }}, \mathrm{b}\right)$ Earthquakes: an instance of $\mathrm{S}_{\text {earthq. }}$

According to [13] fields are geographic objects with a function that maps each point of their geometry to an alphanumeric value. This definition allows for representing field data independently of their implementation (complete/incomplete) (Field Object). Thus, A Field Object extends a Geographic Object with a function that associates each point of the geometry to an alphanumeric value. In this way, a Field Object allows for representing geographic data according to the field and the vector model at the same time ("Independence of implementation" requirement of Table 1).

\section{Definition 3. Field Object}

Let $S_{e}=\left\langle\right.$ geom, field, $\left.\left[a_{1}, \ldots a_{n}\right]\right\rangle$ a Geographic Object Structure. $S_{e}$ is a Field Object Structure if the domain of the attribute field is a set of functions defined on $m$ sub-sets of points of geom having values in an alphanumeric domain $\operatorname{dom}_{\text {field }}: \operatorname{dom}(f i e l d)=\left\{f_{1} \ldots\right.$ $\left.f_{m}\right\}$

An Instance of an Field Object Structure $S_{e}$ is a tuple $\left\langle g, f_{j}\right.$, val $\left(a_{1}\right), \ldots$ val $\left.\left(a_{n}\right)\right\rangle$ where:

- $\forall i \in[1, \ldots n]$ val $\left(a_{i}\right) \in \operatorname{dom}\left(a_{i}\right), g \in \operatorname{dom}(g e o m)$

$-f_{j}: g \rightarrow$ dom $_{\text {field }}$ and $f_{j} \in\left\{f_{1}, \ldots, f_{m}\right\}$

We note 'field support' the input domain of $f_{j}$

\section{Example 2.}

The field object structure representing earthquakes is $S_{\text {earthq }}=\langle$ geom, intensity $\rangle$ where 'geom' is the geometric support, and 'intensity' is a set of functions defined on 'geom' with values in $R$. 'intensity' represents the intensity of the earthquake. An instance of $\mathrm{S}_{\text {earthq }}$ is $\mathrm{t}_{2}=\left\langle\mathrm{p}_{2}, \mathrm{f}_{2}\right\rangle$ where $\mathrm{p}_{2}$ is a geometry and $\mathrm{f}_{2}$ represents the intensity of the earthquake on the 11-1999 in Lombardia. $f_{2}$ is defined on each point of $p_{2}$ with values in $R$, for example $\mathrm{f}_{2}(\mathrm{x} ; \mathrm{y})=12$ (Figure $2 \mathrm{~b}$ ).

By the same way, we can define a field object structure to represent terrain models of Italian regions at different scales by adding to the geographic object $S_{\text {region }}$ the field attribute representing terrain elevation. 


\subsection{Spatio-multidimensional model for field data}

A spatio-multidimensional model organizes data using the concepts of dimensions composed of hierarchies, and facts described by measures. An instance of the spatiomultidimensional model is a hypercube. Section 3.2.1 presents the concepts of dimensions, facts, and measures, and Section 3.2.2 formalizes cuboids.

\subsubsection{Hierarchies and facts}

According to [3] a spatial hierarchy organizes vector objects in a hierarchical way. Formally, a Spatial Hierarchy organizes the Geographic Objects [3] (i.e. vector objects) into a hierarchy structure using a partial order $\leq_{h}$ where $S_{i} \leq_{h} S_{j}$ means that $S_{i}$ is a less detailed level than $S_{j}$. An instance of a hierarchy is a tree $\left(<_{h}\right)$ of instances of Geographic Objects (spatial members). Then, measures are aggregated according to the groups of spatial members defined by the tree $<_{h}$.

Hence, in this work we define a Field Hierarchy as a hierarchy of field objects. For that, we extend the spatial hierarchy by defining a tree $\left(<_{f}\right)$ on the geometric coordinates (field supports) of the spatial members represented by field objects ("Hierarchy on continuous field data" requirement of Table 1). By this way it is possible to visualize field objects at different scales or resolutions (Figure 4). Moreover, the alphanumeric values associated to each point of the field measures are aggregated according to the groups of coordinates of spatial members defined by the tree $<_{f}$. By this way, our model uses the continuous representation of spatial members (Field Objects) to aggregate measure, allowing visualizing field measures at different scales or resolutions (see Figure 6b).

\section{Definition 4. Field Hierarchy}

A Field Hierarchy Structure, $\mathcal{H}_{h}$, is a tuple $\left\langle\mathcal{L}_{h}, L_{h}, \Gamma_{h}, \leq_{h}\right\rangle$ where:

- Lh, $\Gamma_{h}$, are of Field Object Structures, and $\mathcal{L}_{h}$ is a set of Field Object Structures

- $\leq_{h}$ is a partial order defined on $\mathcal{L}_{h}, L_{h}, \Gamma_{h}$ as defined in [3]

An Instance of a Field Hierarchy Structure $\mathcal{H}_{h}$ is two partial orders: $<_{h}$ and $<_{f}$ such that:

$-<_{h}$ is defined on the instances of $\mathcal{L}_{h}, L_{h}, \Gamma_{h}$ as defined in [3]

We note $<_{h}$ 'geographic objects order'

- $<_{f}$ is defined on the field supports of the instances of $\mathcal{L}_{h}, L_{h}, \Gamma_{h}$ such that:

- if $\operatorname{cood}_{i}<_{f} \operatorname{cood}_{j}$ then $S_{i} \leq_{h} S_{j}$, where $\operatorname{cood}_{i}$ belongs to a field support of an

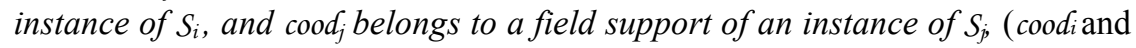
$\operatorname{cood}_{j}$ are geometric coordinates)

- $\forall \operatorname{cood}_{i}$ which does not belong to the field supports of the instances of $\Gamma_{h}, \exists$ one

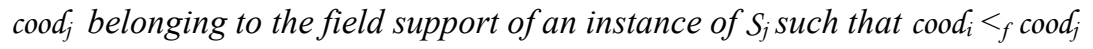

- $\forall \operatorname{cood}_{\mathrm{i}}$ which does not belong to the field supports of the instances of $L_{h}, \exists \operatorname{cood}_{\mathrm{j}}$ belonging to the field support of an instance of $S_{j}$ such that $\operatorname{cood}_{j}<_{f} \operatorname{cood}_{i}$ 
We note $<_{f}$, field objects order'

The set of leafs of the tree represented by $<_{h}$ with root tiare denoted as leafs $\left(\mathcal{H}_{h}, t_{i}\right)$. The set of leafs of the tree represented by $<_{f}$ with root cood ${ }_{i}$ are denoted as leafsFieldSupport $\left(\mathcal{H}_{h}, \operatorname{cood}_{i}\right)$.

\section{Example 3.}

The field hierarchy structure representing the administrative dimension that groups regions into zones is $\mathrm{H}_{\text {location }}=\left\langle\mathcal{L}_{\text {location }}, \mathrm{S}_{\text {region }}, \mathrm{S}_{\text {all_location, }} \leq_{\text {location }}\right\rangle$ where $\mathcal{L}_{\text {location }}=$ $\left\{\mathrm{S}_{\text {zone }}\right\}$ and $\left(\mathrm{S}_{\text {region }} \leq_{\text {location }} \mathrm{S}_{\text {zone }}\right)$. $\mathrm{S}_{\text {region }}$ and $\mathrm{S}_{\text {zone }}$ are the spatial levels of the hierarchy (Figure 3a). An example of instance of $\mathrm{H}_{\text {location }}$ is shown on Figure $3 \mathrm{~b}$ and $3 \mathrm{c}$. We can notice two trees: the geographic objects order that is represented by black lines (Figure 3b), and the field objects order, which is represented by dashed lines (Figure $3 c)$.

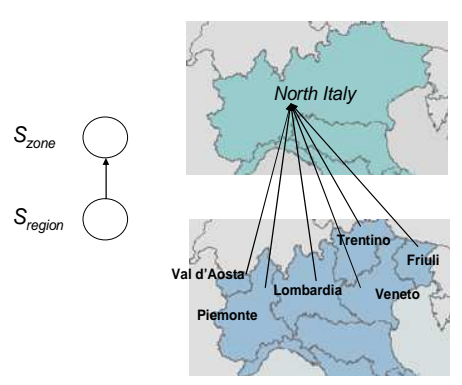

(a)

(b)

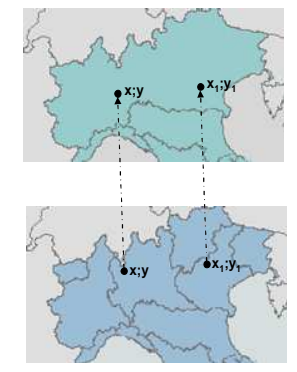

(c)

Figure 3: Field hierarchy grouping regions into zones, a) Schema, b) Hierarchical relationships between geographic objects, c) Hierarchical relationships between geometric coordinates.

\section{Example 4.}

The hierarchy structure representing the terrain models of Italian regions at different resolutions is $\mathrm{H}_{\text {regres }}=\left\langle\mathcal{L}_{\text {regres }}, \mathrm{S}_{\text {region }}, \mathrm{S}_{\text {all_regres, }} \leq_{\text {regres }}\right\rangle$ where $\mathcal{L}_{\text {regres }}=\left\{\mathrm{S}_{\text {regres }}\right\}$ and $\left(\mathrm{S}_{\text {region }}\right.$ $\leq_{\text {regres }} S_{\text {regres }}$ ) (Figure $\left.4 a\right)$. Its instance is shown on Figure $4 b$ and Figure $4 c$. Note that a geometric coordinate at the coarser resolution is associated with a set of geometric coordinates at the most detailed resolution (Figure 4c). For example, this hierarchy can be defined using the bilinear interpolation algorithms used for changing resolution for raster data at different scales. 


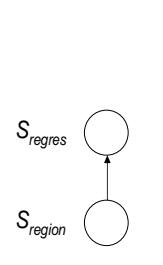

(a)

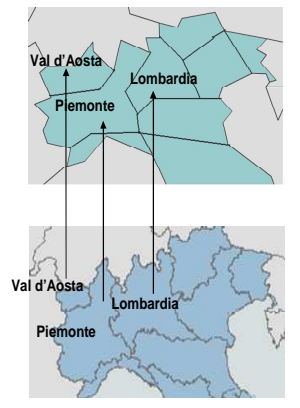

(b)

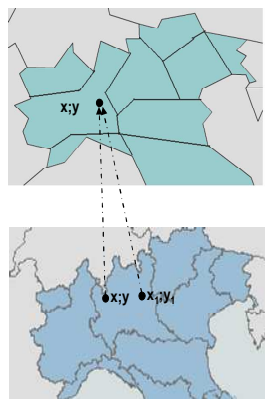

(c)

Figure 4: Field hierarchy representing regions at different scales, a) Schema, b) Hierarchical relationships between geographic objects (geographic objects order), c) Hierarchical relationships between geometric coordinates (field objects order).

Once we have introduced the concepts of measures, and hierarchies for field data, we present the concept of Field Cube. A Field Cube Structure represents the spatiomultidimensional model schema where a field object is used as measure that is analyzed according classical hierarchies and one field hierarchy ("Measures as continuous field data" requirement of Table 1). Note that without losing in generalization, we suppose to have only one spatial dimension and one field measure to simplify the formalism of the model. In the same way, we do not introduce numerical measures as they can be simply represented using numeric attributes as defined in [3]. An instance of a field cube structure represents the fact table or the basic cuboid of the lattice cuboids.

\section{Definition 5. Field Cube}

A Field Cube Structure, $F_{c}$, is a tuple $\left\langle\mathcal{H}_{1}, \ldots \mathcal{H}_{n}\right.$, FieldO6ject $\rangle$ where:

- $\mathcal{H}_{1}$ is a Field Hierarchy Structure (Spatial dimension)

- $\forall i \in[2, \ldots n] \mathcal{H}_{i}$ is a Hierarchy Structure (Dimensions)

- FieldObject is Field Object Structure (Field measure)

An Instance of a Field Cube Structure $F C_{c}, I\left(F C_{c}\right)$, is a set of tuples $\left\{\left\langle t b_{1}, \ldots t 6_{n}, t 6_{f}\right\rangle\right\}$ where:

- $\forall i \in[1, \ldots n] t 6_{i}$ is an instance of the bottom level of $\mathcal{H}_{i}\left(L_{i}\right)$ (Most detailed levels members)

- $t b_{f}$ is an instance of FieldObject (Field measure value)

\section{Example 5.}

The field cube structure of our case study is $\mathrm{FC}_{\text {earthq }}=\left\langle\mathrm{H}_{\text {regtres}}, \mathrm{H}_{\text {time }}, \mathrm{S}_{\text {earthq }}\right\rangle . \mathrm{H}_{\text {regtres }}$ is the field hierarchy (the spatial dimension), $\mathrm{H}_{\text {time }}$ is the temporal dimension, and $\mathrm{S}_{\text {earthq }}$ is the field measure. It allows answering previous formulated query "Where were earthquakes, and what was their intensity per region at different scale (resolutions)?".

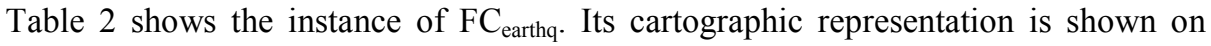
Figure 5. 
Table 2: Instance of $\mathrm{FC}_{\text {earthq. }}$

\begin{tabular}{|c|c|c|}
\hline Reg & Month & Earthq \\
\hline Lombardia & $9-1998$ & $\mathrm{t}_{1}$ \\
\hline Lombardia & $11-1999$ & $\mathrm{t}_{2}$ \\
\hline Piemonte & $11-1999$ & $\mathrm{t}_{6}$ \\
\hline
\end{tabular}

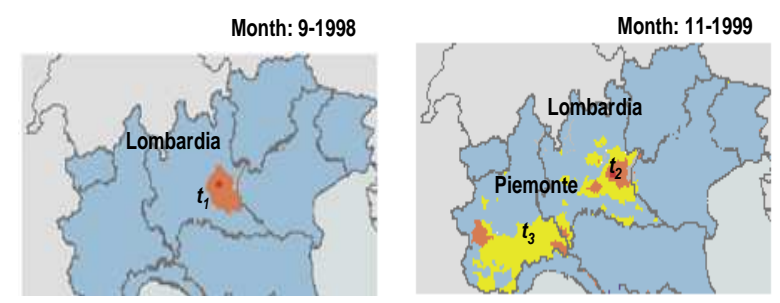

Figure 5: Cartographic representation of the instance of $\mathrm{FC}_{\text {earthq. }}$

\subsubsection{Hypercube}

The instance of the spatio-multidimensional model is a hypercube. A hypercube can be represented as a hierarchical lattice of cuboids [9]. The most detailed cuboid contains detailed measures (basic cuboid). Other cuboids contain aggregated measures. Then, cuboids are represented by levels and (aggregated) measures values (Sec. 3.2.2.2). How field measures are aggregated from fact table data (basic cuboid) to represent non-basic cuboids is presented in Sec. 3.2.2.1.

\subsubsection{Aggregation of field measures}

The aggregation of field measures is defined by means of:

- For the geometric support: spatial aggregation,

- For alphanumeric attributes: alphanumeric aggregations

- For the field attribute:

- Local Map Algebra, or focal/zonal map cubic algebra operator when aggregating on the non-field hierarchies

- Alphanumeric aggregation when aggregating on the field hierarchy

Indeed aggregation of field measures is done in two steps. In the first step we aggregate along the non-field hierarchies, and then along the field hierarchy.

\subsection{Aggregation on non-field hierarchies}

In this section we formalize the geometric and alphanumeric aggregations. 


\section{Definition 6. Spatial aggregation}

Let $G$ the geometric attribute. Its aggregation is defined by means of a function $\mathrm{O}_{G}$ that has as input n geometries of the attribute G, and that returns one geometry:

$$
O_{G}: \operatorname{dom}(G) \times \ldots \times \operatorname{dom}(G) \rightarrow 2^{g} \text { where } g \text { is a subset of the Euclidian Space } R^{2}
$$

\section{Definition 7. Alphanumeric aggregation}

Let $\mathcal{A}$ be an alphanumeric attribute. Its aggregation is defined by means of a function $O_{\mathcal{A}}$ that has in input $n$ values of the attribute $\mathcal{A}$, and that returns one value of the attribute $\mathcal{A}$ :

$$
\mathrm{O}_{\mathcal{A}}: \operatorname{dom}(\mathcal{A}) \times \ldots \times \operatorname{dom}(\mathcal{A}) \rightarrow \operatorname{dom}(\mathcal{A})
$$

On non-field hierarchies, the aggregation of the field attribute is defined by means of a function $\left(O_{F}\right)$ that takes as input a set of functions representing the field attributes values $\left(f_{1} \ldots f_{n}\right)$, and it returns a new function $\left(f_{1 n}\right)$. This function is defined on the field support of $f_{1} \ldots f_{n}$, and the value of each point $\left(f_{1 n}\left(x_{i} y\right)\right)$ is calculated by applying a alphanumeric function $O_{\mathcal{A}}$ to the values of the other functions $\left(O_{F}\left(f_{1}\left(x_{i} y\right) \ldots f_{n}\left(x_{i} y\right)\right)\right.$ ). Then, $O_{F}$ represents a map/map cubic algebra operator that is specialized in local, focal or zonal by means of the $O_{\mathcal{A}}$ function. Indeed, $O_{\mathcal{A}}$ is applied point by point for local map function, or to sets of coordinates defined by the functions $\mathcal{N}$ eightorhood $\left(x_{i} y\right)$ and Zone(FieldObjects, $(x ; y))$ for focal map cubic and zonal map cubic operators respectively (("Aggregation functions as Map/Map Cubic Algebra functions " requirement of Table 1)).

Definition 8. Aggregation of the Field attribute on non-field hierarchies using Map Algebra and Cubic Map algebra functions

Let $F$ be a field attribute, and $f_{1} \ldots f_{n}$ functions of the domain of $F$ with $g$ as field support (without loss of generality, we suppose the $f_{1} \ldots f_{n}$ have the same field support)

$f_{1}: g \rightarrow \operatorname{dom}_{F} \ldots f_{n}: g \rightarrow \operatorname{dom}_{\mathcal{F}}$, and $f_{1} \ldots f_{n} \in \operatorname{dom}(F)$.

Let $\mathrm{O}_{\mathcal{A}}$ be an alphanumeric aggregation

Then, the aggregation of $F$ is defined by means of a function $O_{F}$ that takes as input $f_{1} \ldots f_{n}$, and that returns a function $f_{1 n}$ defined on g and having values in $\operatorname{dom}_{F}\left(f_{1 n}: g \rightarrow\right.$ dom $\left._{F}\right)\left(f_{1 n}=O_{F}\left(f_{1} \ldots f_{n}\right)\right)$ such that:

- using Local operator:

$f_{1 n}(x ; y)=O_{\mathcal{A}}\left(f_{1}(x ; y), \ldots, f_{n}(x ; y)\right)$ for each point $(x ; y)$ of $g$

- using Cubic Focal operator:

$f_{1 n}(x ; y)=O_{\mathcal{A}}\left(f_{1} \quad\left(\mathcal{N}\right.\right.$ eigh6orhood $\left.\left(x_{i} ; y\right)\right) . . f_{n}\left(\mathcal{N}\right.$ eighiborhood $\left.\left.\left(x_{i} ; y\right)\right)\right)$ for each point $(x ; y)$ of $g$ where:

$\mathcal{N}$ eighborhood $(x ; y)$ is a function that returns the neighbourhood points of $(x ; y)$,

- using Cubic Zonal operator:

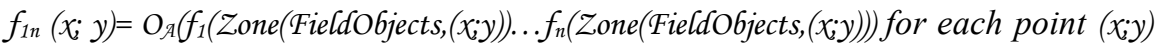
of $g$ where: 
Zone(FieldO6jects, (x;y)) is a function that takes as input a set of Field Objects and a point, and it returns the neighbourhood points of $(x ; y)$ that belong to the zone indentified by the FieldO6jects on this point.

\section{Definition 9. built non-field (Figure 7)}

Let $t b_{f 1}, \ldots t b_{f k}$ and $t_{n f}$ instances of the field object structure $S_{e}=\left\langle\right.$ geom, field, $\left.\left[a_{1}, \ldots a_{m}\right]\right\rangle$ Let $\mathrm{O} \mathcal{N}$, called non-field aggregation mode, a set of aggregation functions:

- $\mathrm{O}_{G}$ the spatial aggregation for geom

- $\mathrm{O}_{1 . .} \mathrm{O}_{m}$ the alphanumeric aggregations for $a_{1}, \ldots a_{m}$

- OF the Map Algebra/Map Cubic Algebra aggregation for field

We say that $t_{n f}$ is built non-field from $t \sigma_{f 1}, \ldots t \sigma_{f \kappa}$ using ONF if:

- $\quad t_{n f . g e o m}=O_{G}\left(t b_{f 1}\right.$. geom, ..., $t b_{f k}$ geom $)$

- $\quad \forall i \in[1, \ldots m] t_{n f} \cdot a_{i .}=O_{i}\left(t \sigma_{f 1}, a_{i}, \ldots, t \sigma_{f k} . a_{i}\right)$

- $\quad t_{n f}$. field $=O_{F}\left(t \sigma_{f 1}\right.$. field,..., $t \widehat{\sigma}_{f k}$. field $)$

\section{Example 6.}

Let an instance of $S_{\text {earthq }} t_{1}=\left\langle p_{1}, f_{1}\right\rangle$ where $p_{1}$ is a geometry and $f_{1}$ represents the intensity of the earthquake on the $9-1998$ in Lombardia. It is defined on each point of $\mathrm{p}_{1}$ with values in $R$. For example $\mathrm{f}_{1}(\mathrm{x} ; \mathrm{y})=10$ (Figure $\left.6 \mathrm{a}\right)$. To aggregate the field attribute intensity on the temporal dimension, we use a cubic focal operator AVG. Therefore the result of the aggregation of $f_{1}$ and $f_{2}$ on $(x ; y)$ by taking into account neighbours of $(x ; y)$ is $f_{3}(x ; y)=((13 * 4+10)+(11 * 4+12)) / 10=11.7$ (we suppose that the values of neighbourhood points of $(x ; y)$ of $t_{1}$ and $t_{2}$ are 10 and 12 respectively) (Figure $6 a)$. We suppose that we apply the geometric union for geometry.

Then, $t_{3}$ is built non-field from $t_{1}$ and $t_{2}$. $t_{3}$ is an aggregated measure of the cuboid defined by the century level of the temporal dimension (see Table 3 ).

Table 3: Instances of the cuboid defined by the century level of the temporal dimension.

\begin{tabular}{|c|c|c|}
\hline Region & Century & Earthq \\
\hline Lombardia & 900 & $\mathrm{t}_{3}$ \\
\hline Piemonte & 900 & $\mathrm{t}_{6}$ \\
\hline
\end{tabular}

Then, as a field measures is mapped also on spatial dimensions, then a particular aggregation must be provided taking into account the field hierarchy in order to allow the visualization of field measures at different resolutions or scales.

\subsection{Aggregation on the field hierarchy}

The aggregated measures of a cuboid defined by coarser spatial levels are the aggregation of the (aggregated) measures of the cuboids defined by non-spatial levels. Definition 10. built field (Figure 7)

Let $t^{1}{ }_{n f}, \ldots, t^{v}{ }_{n f}$ and $t_{f}$ be instances of the field object structure $S_{e}$ 〈geom, field, $\left.\left[a_{1}, \ldots a_{m}\right]\right\rangle$. Let OF, called field aggregation mode, a set of aggregation functions:

- $\mathrm{O}_{G}$ the spatial aggregation for geom 
- $\quad O_{1 . .} O_{m}$ the alphanumeric aggregations for $a_{1}, \ldots a_{m}$

- $\mathrm{O}_{\mathcal{A}}$ the alphanumeric aggregation for field

We say that $t_{f}$ is built field from $t^{1}{ }_{n f}, \ldots, t^{v}{ }_{n f}$ using OF if:

- $t_{f}$.geom $=O_{G}\left(t^{1}{ }_{n f .}\right.$ geom, ..., $\left.t^{v}{ }_{n f . g e o m}\right)$

- $\forall i \in[1, \ldots m] t_{f} \cdot a_{i .}=O_{G}\left(t^{1}{ }_{n f} . a_{i}, \ldots, t^{v}{ }_{n f} \cdot a_{i}\right)$

- $t_{f}$. field $(x ; y)=O_{\mathcal{A}}\left(f^{1}\left(x^{1} ; y^{1}\right), \ldots, f^{m}\left(x^{m} ; y^{m}\right)\right)$ where $f^{1}, \ldots, f^{m}$ belong to $t^{1}{ }_{n f}$. field,$\ldots t^{v}{ }_{n f}$. field, for each point $(x ; y)$ of the field support of field

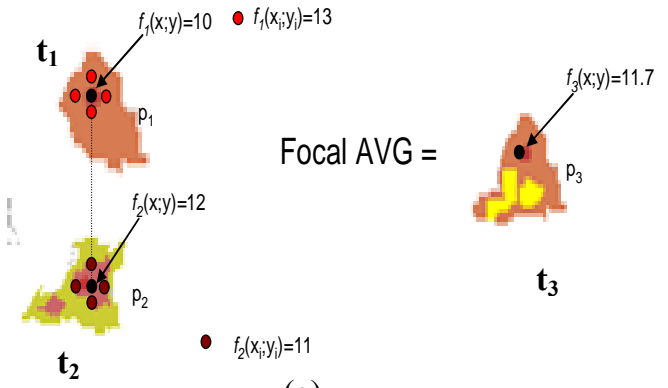

(a)

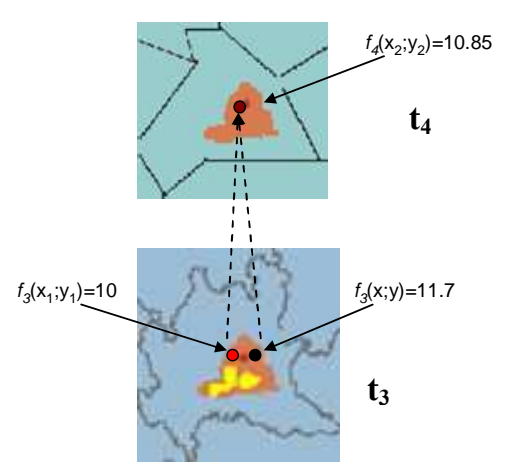

(b)

Figure 6: a) Aggregation on the "intensity" field attribute on the temporal dimension, b) Aggregation on the "intensity" field attribute on the field hierarchy.

\section{Example 7.}

In order to visualize the measure at different resolutions, we aggregate on the Field Hierarchy $\mathrm{H}_{\text {regres }}$ applying the average. Then $\mathrm{f}_{4}(\mathrm{x} ; \mathrm{y})=$ AVG(leavesFieldSupport $\left.\left(\mathrm{H}_{\text {deptres}},\left(\mathrm{x}_{2} ; \mathrm{y}_{2}\right)\right)\right)=\operatorname{AVG}\left(\mathrm{f}_{3}(\mathrm{x} ; \mathrm{y}), \mathrm{f}_{3}\left(\mathrm{x}_{1} ; \mathrm{y}_{1}\right)\right)=(10+11.7) / 2=$ 10.85 (Figure $6 \mathrm{~b})$. Then, $t_{4}$ is built field from $t_{3} . t_{4}$ is an aggregated measure of the cuboid defined by the century and regres levels (see Table 4).

Table 4: Instance of $\mathrm{FC}_{\text {earthq. }}$.

\begin{tabular}{|c|c|c|}
\hline $\begin{array}{c}\text { Regres (region at scale } \\
\mathbf{1 : 1 . 0 0 0 )}\end{array}$ & Century & Earthq \\
\hline Lombardia & 900 & $\mathrm{t}_{4}$ \\
\hline Piemonte & 900 & $\mathrm{t}_{5}$ \\
\hline
\end{tabular}

\subsubsection{Cuboids of field data}

Once described how the measures of the different cuboids are related by aggregation functions, in this section we formalize the concept of cuboid. In particular, a cuboid schema, noted Field View Structure, is composed by a set of levels, a non-field aggregation mode, and a field aggregation mode. An instance of a field view structure is a set of tuples composed of a member for each level and a (aggregated) field measure value. The aggregated field measure value on the spatial 
dimension $\left(t_{f}\right)$ is obtained aggregating measures $\left(t^{1}{ }_{n f} \ldots, t_{n f}^{v}\right)$ obtained after the aggregation on the non-spatial dimensions of detailed measures $\left(t \sigma_{f 1} \ldots, t \sigma_{f k}\right)$ as shown on Figure 7.

\section{Definition 11. Field View}

A Field View Structure $\mathcal{V}_{v}$ is a tuple $\left\langle F C_{c}, \mathcal{L}, O \mathcal{N} F, O F\right\rangle$ where:

- $\quad F C_{c}=\left\langle\mathcal{H}_{1}, \ldots \mathcal{H}_{n}\right.$, FieldO6ject $\rangle$ is a Field Cube Structure (Spatio-multidimensional model schema)

- $\quad \mathcal{L}$ is a tuple $\left\langle S_{1}, \ldots S_{n}\right\rangle$ where $\forall i \in[1, \ldots n] S_{i}$ is a level of $\mathcal{H}_{i}$ (Levels that define the cuboid)

- $\mathrm{ONF}$ is a non field aggregation mode (Aggregation functions used on non-spatial dimensions)

- OF is a field aggregation mode (Aggregation functions used on the spatial dimension)

An Instance of a field view Structure is a set of tuples $\left\{\left\langle t_{1}, \ldots t_{n}, t_{f}\right\rangle\right\}$ where: $\forall i \in[1, \ldots n] t_{i}$ is an instance of $S_{i}$ (Dimensions members)

- $\quad t_{f} i s$ : ((aggregated) field measure on spatial dimension, Figure 7 - see Table 4 for an example)

- $\quad$ an instance of FieldObject

- $\quad$ built field from $t^{1}{ }_{n f}, \ldots, t^{v}$ using OF where ((aggregated) field measures on nonspatial dimensions, Figure 7 - see Table 3 for an example):

- $\quad t_{f}$ field $(x ; y)=O F . O_{\mathcal{A}}\left(f^{1}\left(x^{1} ; y^{1}\right), \ldots, f^{m}\left(x^{m} ; y^{m}\right)\right)$ for each point $(x ; y)$ of its field support where:

- $\left(x^{1} ; y^{1}\right), \ldots,\left(x^{m} ; y^{m}\right)$ belong to leafsFieldSupport $\left(\mathcal{H}_{1},(x ; y)\right)$

- $\quad f^{1}, \ldots, f^{m}$ belong to $t^{1}$ ff. field,..., $t^{v}{ }_{n f}$. field

- Each $t_{n f}$ is built non field from $t b_{f 1}, \ldots, t b_{f k}$ using ONF where (Non aggregated field measures, Figure 7 - see Table 2 for an example):

- $t b_{f 1}, \ldots, t b_{f k}$ are the measure values of the tuples of $I\left(F C_{c}\right)\left\langle t b_{1} i_{1}\right.$ $\left.t 6^{1}{ }_{2} \ldots t 6^{1}{ }_{n}, t b_{f 1}\right\rangle, \ldots,\left\langle t 6_{1}{ }_{1}, t \sigma^{k}{ }_{2} \ldots t \delta^{k_{n}}, t 6_{f k}\right\rangle$ where:

- $\forall i \in[2, \ldots n] t 6^{1}{ }_{i} \ldots t 6^{\kappa_{i}}=\operatorname{leafs}\left(\mathcal{H}_{i}, t_{i}\right)$

- $t b_{1}{ }_{1}$ belongs to leafs $\left(\mathcal{H}_{1}, t_{1}\right)$

Example 8.

The Field View Structure representing earthquakes per region at the scale 1:10000 and per century is $\mathrm{V}_{\text {earthq }}=\left\langle F C_{\text {earth }},\left\langle S_{\text {century }}, S_{\text {regres }}\right\rangle\right.$, $\langle$ Union, Focal-Avg $\rangle,\langle$ Union, Avg $\left.\rangle\right\rangle$. Table 4 shows its instance.

\section{Conclusion and future work}

Integration of spatial data into multidimensional models leads to the concept of SOLAP. SOLAP models exploit the discrete representation of spatial data. Few works integrate continuous field data into dimensions and measures. In this paper, motivated by the relevance of a formal representation of SOLAP data, we provide a multidimensional model that considers field data independently form their 
implementation, as measures and dimensions. In particular we provide a unique data model for vector and field data (Geographic and Field Objects). We provide a formal representation of the spatio-multidimensional model schema (Field Cube: Field Hierarchy and Field Measures) and the associated hypercube's cuboids (Field View). Actually, we are working on the formal definition of SOLAP operators that allows the navigation between the cuboids (roll-up/drill-down), and slicing the cuboids (slice). We plan to work on the implementation of the model in a ROLAP architecture. This implies the definition of: (i) query languages for OLAP server [18] for field data [12], (ii) indexes [20] and pre-aggregation techniques [19] for spatial data warehouses using field dimensions and measures, and (iii) interactive field maps [17] for SOLAP clients.

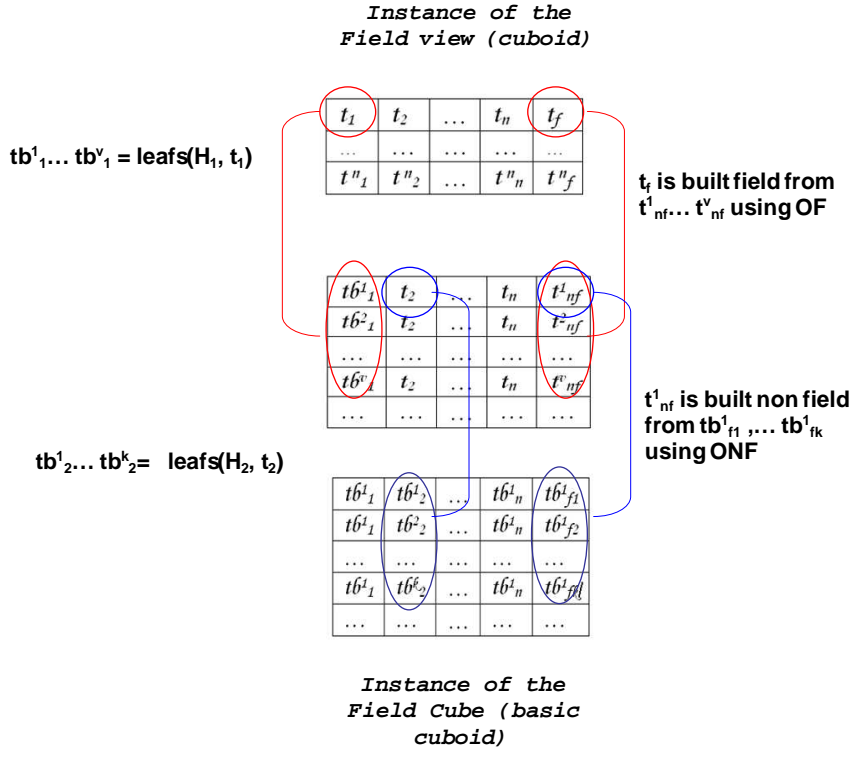

Figure 7: Instance of a Field View Structure

\section{References}

[1] Ahmed, T., Miquel, M.: Multidimensional Structures Dedicated to Continuous Spatiotemporal Phenomena. In: 22th British National Conference on Databases. LNCS, vol. 3567 pp. 29-40. Springer, Heidelberg (2005)

[2] Bédard, Y., Han, J.: Fundamentals of Spatial Data Warehousing for Geographic Knowledge Discovery. Geographic Data Mining and Knowledge Discovery. Taylor \& Francis, New York, USA (2009)

[3] Bimonte, S., Gensel, J., Bertolotto, M.: Enriching Spatial OLAP with Map Generalization: a Conceptual Multidimensional Model. In: IEEE International Workshop on Spatial and Spatiotemporal Data Minin, pp. 332 - 34. IEEE CS Press (2008)

[4] Bimonte, S., Tchounikine, A., Miquel, M., Pinet, F.: When Spatial Analysis Meets OLAP: Multidimensional Model and Operators. International Journal of DataWarehousing and Mining (to appear) 
[5] Câmara, G., De Freitas, U., Cordeiro, J.: Towards an algebra of geographical fields. In: Brazilian Symp. on computer graphics and image processing, Anais, Curitiba, pp. 205-212 (1994)

[6] Di Martino, S., Bimonte, S., Bertolotto, M., Ferrucci; F.: Integrating Google Earth within OLAP Tools for Multidimensional Exploration and Analysis of Spatial Data. In: ICEIS 2009. LNBIP, vol. 24, pp. 940-951. Springer, Heidelberg (2009)

[7] Franklin, C.: An Introduction to Geographic Information Systems: Linking Maps to databases. Database, 15(2), 13-21 (1992)

[8] Gutierrez, A., Baumann, P.: Modeling Fundamental Geo-Raster Operations with Array Algebra. In: IEEE International Workshop on Spatial and Spatiotemporal Data Mining, pp. 607-612. IEEE CS Press (2007)

[9] Harinarayan, V., Rajaraman, A., Ullman, J. D.: Implementing Data Cubes Efficiently. In: ACM SIGMOD International Conference on Management of Data, pp. 205-216. ACM Press, New York (1996)

[10] Kemp, K.: Environmental Modeling with GIS: A Strategy for Dealing with Spatial Continuity. Technical Report 93-3. National Center for Geographic Information and Analysis, University of California, Santa Barbara, USA (1993)

[11] Kimball, R.: The Data Warehouse Toolkit: Practical Techniques for Building Dimensional Data Warehouses. John Wiley \& Sons, New York, USA (1996).

[12] Laurini, R., Gordillo, S.: Field Orientation for Continuous Spatio-temporal Phenomena. In: International Workshop on Emerging Technologies for Geo-based Applicatons. pp. 77-101 Swiss Federal Institute of Technology, Lausanne (2000)

[13] Ledoux, H., Gold., C.M.: A Voronoi-based Map Algebra. In: 12th International Symp. on Spatial Data Handling, pp. 117-131. Springer, Heidelberg (2006)

[14] Malinowski, E., Zimányi, E.: Advanced Data Warehouse Design From Conventional to Spatial and Temporal Applications. Springer, Heidelberg (2008)

[15] McHugh, R.: Intégration De La Structure Matricielle Dans Les Cubes Spatiaux. Université Laval (2008)

[16] Mennis, J., Viger, R., Tomlin, C.D.: Cubic Map Algebra functions for spatio-temporal analysis. Cartography and Geographic Information Systems, 30(1), 17-30 (2005)

[17] Plumejeaud, C., Vincent, J., Grasland, C., Bimonte, S., Mathian, H., Guelton, S., Boulier, J., Gensel, J.: HyperSmooth, a system for Interactive Spatial Analysis via Potential Maps. In: International Symposium on Web and Wireless Geographical Information Systems. LNCS, vol. 5373, pp. 4-16. Springer, Heidelberg (2008)

[18] Silva, J., Castro Vera, A.S., Oliveira, A.G., Fidalgo, R., Salgado, A.C., Times, V.C.: Querying geographical data warehouses with GeoMDQL. In: Brazilian Symposium on Databases, pp. 223-237 (2007)

[19] Stefanovic, N., Han, J., Koperski, K.: Object-Based Selective Materialization for Efficient Implementation of Spatial Data Cubes. IEEE Transactions on Knowledge and Data Engineering, 12 (6), 938-958 (2000)

[20] Tao, Y., Papadias, D.: Historical spatio-temporal aggregation. ACM Trans. Inf. Syst., 23(1), 61-102 (2005)

[21] Timpf, S., Frank, A. U.: Using hierarchical spatial data structures for hierarchical spatial reasoning. In: Spatial Information Theory - A Theoretical Basis for GIS.LNCS, vol. 1329, pp 69-83. Springer, Heidelberg (1992)

[22] Tomlin, C.D.: Geographic Information Systems and Cartographic Modeling. Prentice Hall, Englewood Cliffs, NJ (1990)

[23] Vaisman, A., Zimányi, E.: A multidimensional model representing continuous fields in spatial data warehouses. In: 17th ACM SIGSPATIAL International Symp. on Advances in Geographic Information Systems, pp. 168-177. ACM Press, New York, USA (2009) 